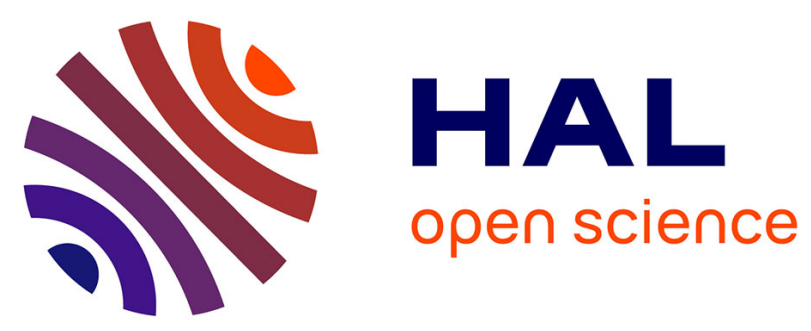

\title{
Strain Gauges Based 3D Shape Monitoring of Beam Structures Using Finite Width Gauge Model
}

Pierre-Loup Schaefer, Guilhaume Barrier, Grégory Chagnon, Thierry Alonso, Alexandre Moreau-Gaudry

\section{- To cite this version:}

Pierre-Loup Schaefer, Guilhaume Barrier, Grégory Chagnon, Thierry Alonso, Alexandre MoreauGaudry. Strain Gauges Based 3D Shape Monitoring of Beam Structures Using Finite Width Gauge Model. Experimental Techniques, 2019. hal-02017091

\section{HAL Id: hal-02017091 https://hal.science/hal-02017091}

Submitted on 17 Feb 2019

HAL is a multi-disciplinary open access archive for the deposit and dissemination of scientific research documents, whether they are published or not. The documents may come from teaching and research institutions in France or abroad, or from public or private research centers.
L'archive ouverte pluridisciplinaire HAL, est destinée au dépôt et à la diffusion de documents scientifiques de niveau recherche, publiés ou non, émanant des établissements d'enseignement et de recherche français ou étrangers, des laboratoires publics ou privés. 


\title{
Strain Gauges Based 3D Shape Monitoring of Beam Structures Using Finite Width Gauge Model
}

\author{
P.-L. Schaefer ${ }^{1}$. G. Barrier ${ }^{1}$. G. Chagnon $^{1}$ - T. Alonso $^{1}$ - A. \\ Moreau-Gaudry ${ }^{1}$
}

Received: date / Accepted: date

\begin{abstract}
This paper presents a new approach validated experimentally to reconstruct with strain gauges the deformed shape of a straight beam with circular cross section. It is based on a novel beam-specific strain gauge model that improves the strain measurement by taking into account the width of the gauges. These improved strain measurements are used by a $3 \mathrm{D}$ finite strain large displacement beam shape reconstruction method to recover the deformed shape iteratively. The whole reconstruction approach has been validated experimentally with 3D deformations of a beam instrumented with strain gauges. Results show that the strain gauge model developed improves reconstruction accuracy and that beam reconstruction can be achieved effectively.
\end{abstract}

Keywords Beam monitoring - 3D reconstruction . Shape reconstruction · Strain sensor - Strain measurement

\section{Pierre-Loup Schaefer}

E-mail: pierre-loup.schaefer@univ-grenoble-alpes.fr

Guillaume Barrier

E-mail: barrier.guillaume@gmail.com

Grégory Chagnon

E-mail: gregory.chagnon@univ-grenoble-alpes.fr

Thierry Alonso

E-mail: thierry.alonso@univ-grenoble-alpes.fr

Alexandre Moreau-Gaudry

E-mail: alexandre.moreau-gaudry@univ-grenoble-alpes.fr

1 Univ. Grenoble Alpes, CNRS, CHU Grenoble Alpes, Institute of Engineering Univ. Grenoble Alpes, TIMC-IMAG, F-38000 Grenoble, France

\section{Introduction}

Real-time shape monitoring of a deformed material structure is an important problem with various domains of applications, such as in the construction $[1,2,3]$ or the medical field $[4,5,6]$. A simple and straightforward way to monitor shape consists in measuring directly the displacement of the material structure by using technologies based on electronics and optics, such as deflection gauge, CCD camera or laser scanner [7, 8]. Nevertheless, this type of direct monitoring can be challenging in some cases due to instrumentation constraints and hidden or unreachable areas [9]. Structure shape monitoring can then be achieved indirectly by reconstructing the shape from the structure characteristics, such as strain.

The topic of using strain measures for beam shape monitoring has been widely covered in the litterature on both theoretical [10, 11, 12, 13, 14] and applied aspects, such as needles deflections during medical intervention $[4,5,15]$ or bridge deformation over time [16]. The strain measures are acquired through technologies such as strain gauges [10, 13, 17], or fiber Bragg gratings $[16,18,19,20,21]$ and are used to obtain deformations of the beam. Depending on the orientations of the sensors these measures can be used to recover the different deformations of the beam such as bending [12], torsion [22] and shearing and elongation [14]. The strain measures are thus used as inputs in a reconstruction method to obtain the structure deformed shape. The reconstruction methods proposed in the literature are either two dimensional $[10,11,16,17,18,23]$ or three dimensional $[12,14,19,20,21]$ depending on the deformation hypothesis made.

The goal of this paper is to propose a new approach to reconstruct the deformed shape of a circular straight 


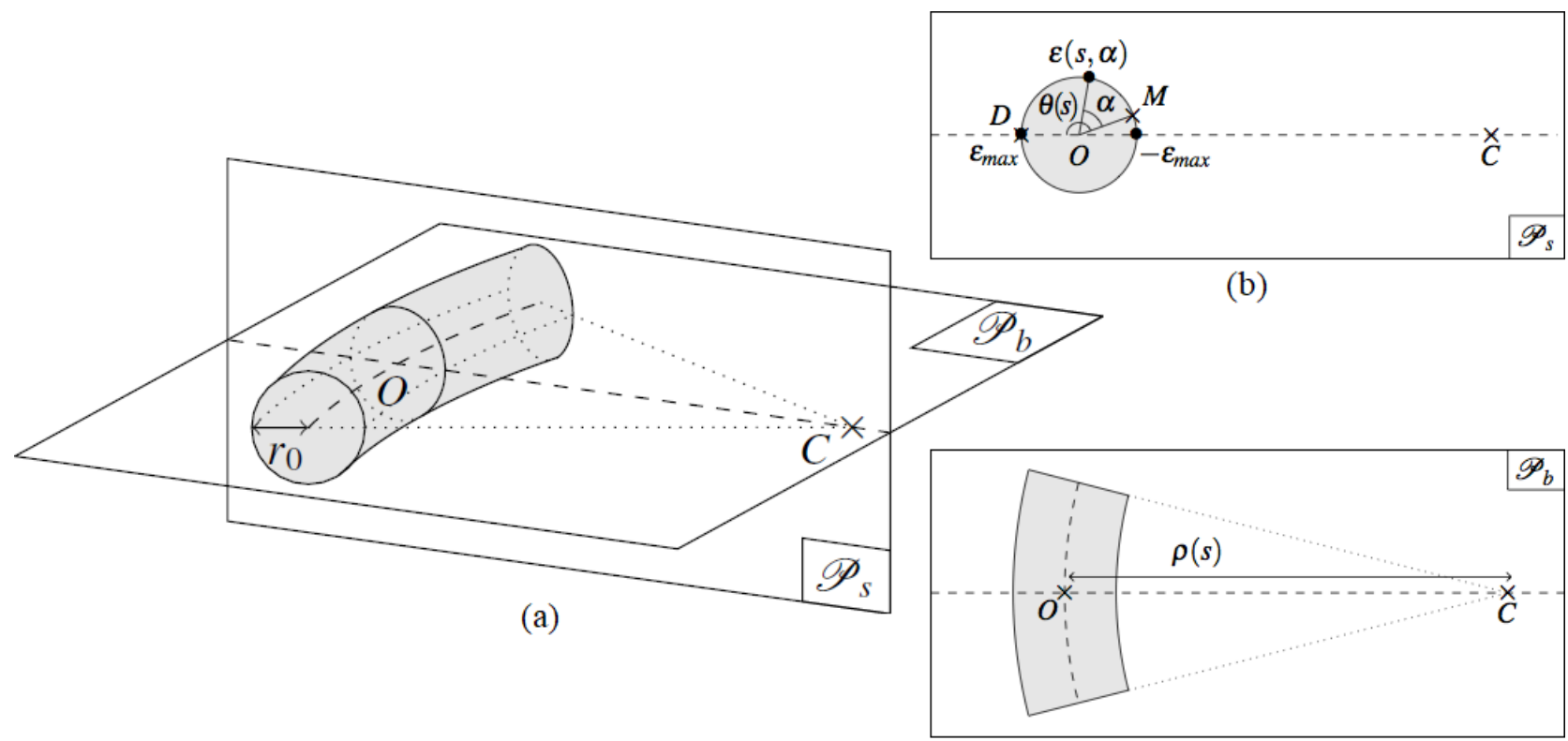

(c)

Fig. 1 Deformation of a beam element

(a) The plane of bending $\mathcal{P}_{b}$ is the osculating plane to the beam's neutral axis at point $O$ and the section plane $\mathcal{P}_{s}$ is the plane orthogonal to $\mathcal{P}_{b}$ containing $O$ and the center of the osculating circle $C$.

(b) Section plane $\mathcal{P}_{s}$ : The bending angle $\theta(s)$ related to a reference material point on the surface of the cross section is the angle between this point and the plane of bending $\mathcal{P}_{b}$. The maximum and minimum strains are noted $\varepsilon_{\max }$ and $-\varepsilon_{\max }$.

(c) Plane of bending $\mathcal{P}_{b}$ : The radius of curvature $\rho(s)$ is the distance between $O$ and $C$.

beam undergoing 3D deformations and to qualify experimentally its accuracy. Section 2 introduces a novel beam-specific strain gauge model and a 3D beam shape reconstruction method based on beam theory. Section 3 presents a test bench composed of a beam instrumented with strain gauges for reconstruction purpose. The procedures and informations on the deformations experiments are described in Section 4. The reconstructed shapes are then compared with the real beam shapes. Section 5 and 6 present and discuss the results of the reconstruction experiments. Finally, Section 7 provides conclusion and plans for future work.

\section{Models}

This section introduces a novel model of strain gauge taking into account its width and a 3D beam shape reconstruction method based on Reissner's finite strain large displacement beam theory [24].

\subsection{Characterization of Beam Deformation from Strain Gauges}

\subsubsection{Beam deformation}

Let $r_{0}$ be the radius of the beam and $O$ a point of the beam's neutral axis of curvilinear abscissa $s$. The deformation characteristics of the beam at point $O$ are presented in figure 1 .

The osculating plane to the beam's neutral axis at point $O$ is noted $\mathcal{P}_{b}$ and called the plane of bending. The distance between $O$ and the center of the osculating circle $C$ is the radius of curvature $\rho(s)$ as shown in figure 1(c). The curvature of the neutral axis at point $O$ is defined as the inverse of the radius of curvature and is noted $\kappa(s)=1 / \rho(s)$. The plane orthogonal to $\mathcal{P}_{b}$ containing $O$ and $C$ is noted $\mathcal{P}_{s}$ and called the section plane. The intersection of the beam with the section plane $\mathcal{P}_{s}$ then defines the cross section of the beam at point $O$ as illustrated in figure 1(b). The rigid cross section thus remains orthogonal to the neutral axis, the shearing deformation is therefore not considered. The intersection between the surface of the cross section and the bending plane which does not belong to the segment $O C$ is noted $D$. Let $M$ be a reference material point 


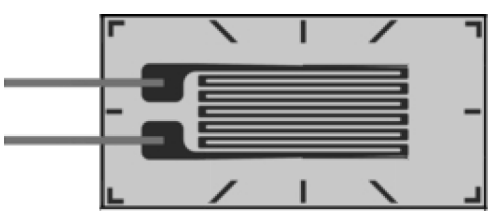

Fig. 2 Scheme of a resistance strain gauge whose resistance value changes with the gauge deformation. The strain measured is the strain in the direction of the gauge length.

on the surface of the cross section. The angle $\widehat{M O D}$ associated to $M$ is noted $\theta(s)$ and called bending angle. The axial strain at the surface of the beam at the angle position $\alpha$ related to $M$ is then noted $\varepsilon(s, \alpha)$. Under the hypothesis of linear elastic deformations, the axial strain $\varepsilon(s, \alpha)$ can be expressed with the curvature $\kappa(s)$, the bending angle $\theta(s)$ and $\delta(s)$ the bias due to other deformations than bending $[5,25]$ :

$\varepsilon(s, \alpha)=r_{0} \kappa(s) \cos (\theta(s)-\alpha)+\delta(s)$

The torsion, tensile and compressive deformations of the beam are not taken into account. It should be noted that the value of strain $\varepsilon(s, \alpha)$ in (1) is independent from the choice of the material point $M$.

\subsubsection{Finite Width Strain Gauge Model}

Beam shape monitoring with strain gauges consists of reconstructing the shape of the beam from the acquired data of the strain gauges. A strain gauge is a sensor which provides a strain measure $\varepsilon^{*}$ of the strain $\varepsilon$ of the surface where it is fixed. This device is composed of an electrical resistance, as illustrated in figure 2 . The deformation of a gauge has for effect to change its electrical resistance value which can be measured. The gauge strain measure $\varepsilon^{*}$ is the mean of the strain conditions existing under its surface [26]. In the case of beam monitoring with strain gauges placed parallel to the beam, when the length of the strain gauge is small compared to the length of the beam, the variation of the axial strain along the length of the gauge is neglected. Moreover, when the plane of bending is orthogonal to the gauge the axial strain is constant along the width of the beam, such as in bridge deformation monitoring [16]. Consequently, as the strain field is supposed uniform under the strain gauge, the averaging by the gauge is ignored $[16,17]$. For some circular beam shape monitoring, such as needle shape reconstruction, the strain sensor width is not taken into account and the strain measurements are supposed to be point-related $[27,15,5]$. We name that model Infinitesimal Width Strain Gauge model (IW). Consequently, the expression

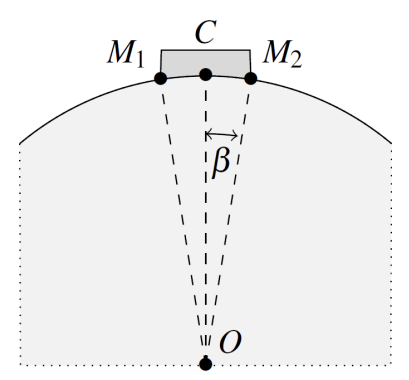

(a)

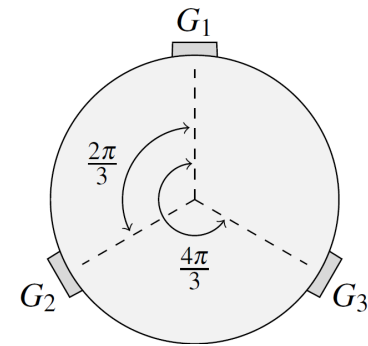

(b)
Fig. 3 Strain gauge fixed on the surface of the beam. (a) The segment $O C$ is the radius between the center of the cross section of the beam and the center of the gauge. The segment $O M_{i}$ is the radius between the center of the cross-section and the side of the gauge. The angle between $O C$ and $O M_{i}$ is called the semi-angle of the gauge and is noted $\beta$.

(b) Sensor triplets composed of strain gauges $G_{1}, G_{2}$, $G_{3}$ placed on a beam cross section with a $120^{\circ}$ angle between them. The angular position $\alpha_{i}$ of the gauge $G_{i}$ on the cross section is expressed relatively to the reference position of the gauge $G_{1}$. Thus $\alpha_{1}=0, \alpha_{2}=$ $\frac{2 \pi}{3}$ and $\alpha_{3}=\frac{4 \pi}{3}$.

of the strain measure $\varepsilon_{I W}^{*}$ of a strain gauge located at angle $\alpha_{g}$ using the IW model is:

$$
\begin{aligned}
\varepsilon_{I W}^{*}\left(s, \alpha_{g}\right) & =\varepsilon\left(s, \alpha_{g}\right) \\
& =r_{0} \kappa(s) \cos \left(\theta(s)-\alpha_{g}\right)+\delta(s)
\end{aligned}
$$

Nevertheless, the dependance of $\varepsilon$ to the variable $\alpha$ in (1) shows that the axial strain on the surface of the beam is not constant along the width of the strain gauge. In order to obtain an accurate gauge model, we developed the Finite Width Strain Gauge model (FW), which takes into account the width of the gauges. The gauge width is then characterized by its semi-angle $\beta$ defined in figure $3 \mathrm{a}$. By using the strain field relation provided by Schajer [28] with the hypothesis that the axial strain is constant along the length of the gauge, the strain measure $\varepsilon_{F W}^{*}$ with the FW model corresponds to the averaging of the strain field on the width 
of the gauge:

$$
\begin{aligned}
\varepsilon_{F W}^{*}\left(s, \alpha_{g}\right) & =\frac{1}{2 \beta r_{0}} \int_{\alpha_{g}-\beta}^{\alpha_{g}+\beta} \varepsilon(s, \alpha) r_{0} d \alpha \\
& =\frac{1}{2 \beta} \int_{\alpha_{g}-\beta}^{\alpha_{g}+\beta}\left[r_{0} \kappa(s) \cos (\theta(s)-\alpha)+\delta(s)\right] d \alpha \\
& =\frac{r_{0} \kappa(s)}{2 \beta} \int_{\alpha_{g}-\beta}^{\alpha_{g}+\beta} \cos (\theta(s)-\alpha) d \alpha+\delta(s) \\
& =r_{0} \frac{\sin (\beta)}{\beta} \kappa(s) \cos \left(\theta(s)-\alpha_{g}\right)+\delta(s) \\
& =r_{0} \operatorname{sinc}(\beta) \kappa(s) \cos \left(\theta(s)-\alpha_{g}\right)+\delta(s)
\end{aligned}
$$

Consequently, the expression of the strain measure $\varepsilon^{*}$ with the IW model (3) and the FW model (8) differs by the term $\operatorname{sinc}(\beta)$ which results from the hypothesis of the strain averaging. The estimations of curvature, bending angle and bias are noted $\kappa_{I W}, \theta_{I W}$ and $\delta_{I W}$ using IW model and $\kappa_{F W}, \theta_{F W}$ and $\delta_{F W}$ using FW model. Equations (3) and (8) give the following relations:

$\kappa_{F W}=\frac{1}{\operatorname{sinc}(\beta)} \kappa_{I W}, \quad \theta_{F W}=\theta_{I W}, \quad \delta_{F W}=\delta_{I W}$

Thus, the expressions of bending angle $\theta$ and bias $\delta$ are identical with both models. On the contrary, the expression of the curvature is different and its value is multiplied by a factor of $1 / \operatorname{sinc}(\beta)$ with the $\mathrm{FW}$ model. Consequently, the use of IW model leads to an underassessment of the curvature compared to the FW model. The percent error of the curvature estimation between FW and IW models is presented in figure 4 . It should be noted that when $\beta \rightarrow 0, \operatorname{sinc}(\beta) \rightarrow 1$ and then $\varepsilon_{F W}^{*} \rightarrow \varepsilon_{I W}^{*}$ and $\kappa_{F W} \rightarrow \kappa_{I W}$. Therefore, the FW model converges to the IW model when $\beta$ tends to 0 .

\subsubsection{Beam Instrumentation with Strain Gauges}

The strain $\varepsilon$ on the surface of a cross section of the beam depends of the three unknowns which are the curvature $\kappa$, the bending angle $\theta$ and the bias $\delta$ as shown in (8). In some case, the positions of the loads on a structure are restricted or can be anticipated. It is then possible to formulate hypothesis on the direction of deformations which allow to reduce the number of strain gauges necessary for shape reconstruction [16]. As no restrictive hypothesis have been formulated on the direction of the deformation of the beam, it is thus necessary to place three strain gauges per instrumented cross section. The strain gauges are placed by groups of three with a $120^{\circ}$ between them $[27,15,5]$, as shown in figure $3 \mathrm{~b}$. This configuration is named triplet in the rest of the paper.

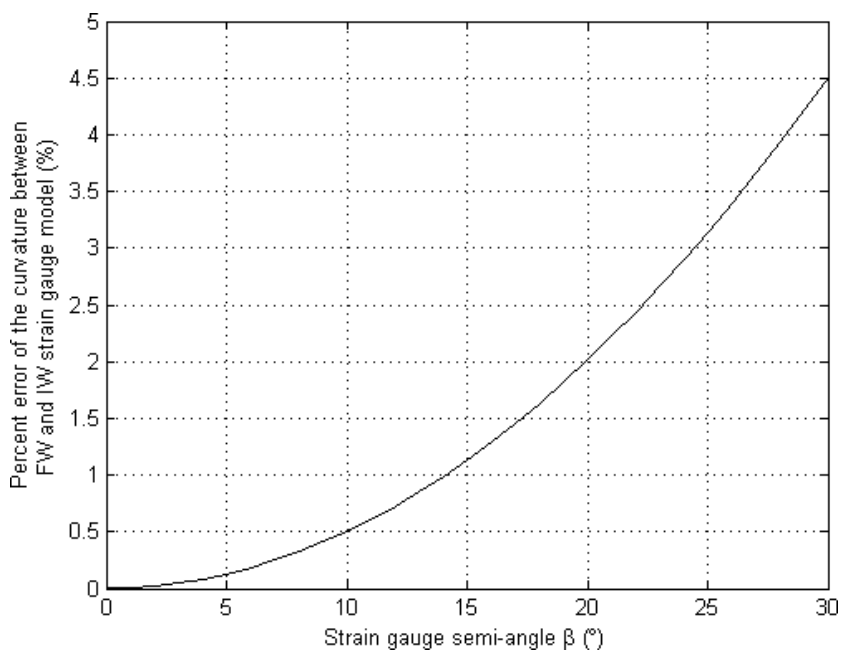

Fig. 4 Percent error between the curvature values obtained with IW and FW models.

By orienting the strain gauges parallely to the beam, the axial surface strain can be measured. The sensor triplet located on the cross section is then composed of strain sensors $G_{1}, G_{2}$ and $G_{3}$ whose respective local axial strain measures are $\varepsilon_{1}^{*}, \varepsilon_{2}^{*}$ and $\varepsilon_{3}^{*}$. For the three strain gauges of a triplet, the FW model gives the following system:

$\varepsilon_{1}^{*}=r_{0} \operatorname{sinc}(\beta) \kappa \cos (\theta)+\delta$

$\varepsilon_{2}^{*}=r_{0} \operatorname{sinc}(\beta) \kappa \cos \left(\theta-\frac{2 \pi}{3}\right)+\delta$

$\varepsilon_{3}^{*}=r_{0} \operatorname{sinc}(\beta) \kappa \cos \left(\theta-\frac{4 \pi}{3}\right)+\delta$

Resolution of (10) gives the following values of curvature $\kappa$, bending angle $\theta$ and bias $\delta$ :

$$
\begin{aligned}
\kappa & =\frac{1}{\operatorname{sinc}(\beta)} \times \frac{C}{3 r_{0}} \\
\cos (\theta) & =\frac{2 \varepsilon_{1}^{*}-\varepsilon_{2}^{*}-\varepsilon_{3}^{*}}{C} \\
\sin (\theta) & =\frac{\sqrt{3}\left(\varepsilon_{2}^{*}-\varepsilon_{3}^{*}\right)}{C} \\
\delta & =\frac{\varepsilon_{1}^{*}+\varepsilon_{2}^{*}+\varepsilon_{3}^{*}}{3}
\end{aligned}
$$

where

$C=\sqrt{2}\left(\left(\varepsilon_{1}^{*}-\varepsilon_{2}^{*}\right)^{2}+\left(\varepsilon_{2}^{*}-\varepsilon_{3}^{*}\right)^{2}+\left(\varepsilon_{1}^{*}-\varepsilon_{3}^{*}\right)^{2}\right)^{\frac{1}{2}}$

\subsection{Beam Shape Reconstruction}

The values of the curvature and the bending angle can be obtained at each triplet location using (11), (12) and (13). The interpolation of those values gives the 
curvature estimate $\kappa$ and the bending angle estimate $\theta$ along the whole length of the needle.

Let $\left(\mathbf{T}, \mathbf{N}_{\mathbf{1}}, \mathbf{N}_{\mathbf{2}}\right)$ be the convected material frame of the beam with $\mathbf{T}$ the row vector of the tangent of the beam neutral axis and $\mathbf{N}_{\mathbf{1}}$ and $\mathbf{N}_{\mathbf{2}}$ two material row vectors of the beam. The evolution of the frame along the length of the beam depends on the curvature $\kappa$ and the bending angle $\theta$ [29]:

$\mathbf{Y}^{\prime}(s)=\mathbf{A}(s) \mathbf{Y}(s)$

$\mathbf{Y}(0)=\mathbf{Y}_{0}$

where:

$\mathbf{Y}(s)=\left[\begin{array}{c}\mathbf{T}(s) \\ \mathbf{N}_{\mathbf{1}}(s) \\ \mathbf{N}_{\mathbf{2}}(s)\end{array}\right]$

$\mathbf{A}(s)=\kappa(s)\left[\begin{array}{ccc}0 & \cos \theta(s)-\sin \theta(s) \\ -\cos \theta(s) & 0 & 0 \\ \sin \theta(s) & 0 & 0\end{array}\right]$

The expression of $\mathbf{A}$ in (19) shows that the frame $\left(\mathbf{T}, \mathbf{N}_{\mathbf{1}}, \mathbf{N}_{\mathbf{2}}\right.$ ) satisfies parallel transport properties. Such frames are called rotation-minimizing frames and are characterized by their stability and their absence of singularities compared to other frames such as SerretFrenet [30]. Equation (17) will be resolved with the iterative method called Local Coordinates Approach [31]. The iterative step of the method is based on the Magnus expansion [32] which gives an exponential representation of the solution of first order matrix differential equation such as (17). The interval of resolution is noted $\left[s_{i}, s_{i+1}\right]$ with $s_{i+1}=s_{i}+h, h$ being the step of the method. The approximation of $\mathbf{Y}\left(s_{i}\right)$ and $\mathbf{Y}\left(s_{i+1}\right)$ are noted $\mathbf{Y}_{i}$ and $\mathbf{Y}_{i+1}$. The matrix of initial condition $\mathbf{Y}_{0}$ contains the coordinates of the moving frame at length $s_{0}$ and is composed of the row vectors $\mathbf{T}\left(s_{0}\right)$, $\mathbf{N}_{\mathbf{1}}\left(s_{0}\right)$ and $\mathbf{N}_{\mathbf{2}}\left(s_{0}\right)$. Here the length $s_{0}$ corresponds to the clamped extremity of the beam.

$\mathbf{Y}_{0}=\left[\begin{array}{c}\mathbf{T}\left(s_{0}\right) \\ \mathbf{N}_{\mathbf{1}}\left(s_{0}\right) \\ \mathbf{N}_{\mathbf{2}}\left(s_{0}\right)\end{array}\right]$

The truncation of the Magnus expansion and the approximation by midpoint rule gives the following iterative step of order 2 [31]:

$\mathbf{Y}_{i+1}=\exp \left(h \mathbf{A}\left(s_{i}+\frac{h}{2}\right)\right) \mathbf{Y}_{i}$

Equation (21) contains a matrix exponential. Using the formula about exponential map in $S O(3)$ [33] gives the following expression:

$\exp \left(h \mathbf{A}\left(s_{i}+\frac{h}{2}\right)\right)=\left[\begin{array}{ccc}c_{1} & s_{1} c_{2} & -s_{1} s_{2} \\ -s_{1} c_{2} c_{1}+\lambda s_{2}^{2} & \lambda c_{2} s_{2} \\ s_{1} s_{2} & \lambda c_{2} s_{2} & c_{1}+\lambda c_{2}^{2}\end{array}\right]$ where

$$
\begin{aligned}
& c_{1}=\cos \left(h \kappa\left(s_{i}+\frac{h}{2}\right)\right) \\
& s_{1}=\sin \left(h \kappa\left(s_{i}+\frac{h}{2}\right)\right) \\
& c_{2}=\cos \left(\theta\left(s_{i}+\frac{h}{2}\right)\right) \\
& s_{2}=\sin \left(\theta\left(s_{i}+\frac{h}{2}\right)\right) \\
& \lambda=1-h \kappa\left(s_{i}+\frac{h}{2}\right)
\end{aligned}
$$

Thus the set $\left(\mathbf{Y}_{i}\right)_{i=0, . ., n}$ is obtained by using (21) iteratively on the discretization of the beam length $\left(s_{i}\right)_{i=0, . ., n}$. It contains the coordinates of the tangents of the beam neutral axis $\left(\mathbf{T}_{i}\right)_{i=0, . ., n}$. Let $\left(\mathbf{M}_{i}\right)_{i=0, . ., n}$ be the set of points of the neutral axis of the beam for the discretization $\left(s_{i}\right)_{i=0, . ., n}$ with $\mathbf{M}_{i}$ the approximation of the position of the neutral axis $\mathbf{M}\left(s_{i}\right)$ for $i=1, . ., n$ and $\mathbf{M}_{0}$ the coordinates of the beam extremity. This set can be calculated iteratively using the tangents obtained previously:

$\mathbf{M}_{i+1}=\mathbf{M}_{i}+h \mathbf{T}_{i}$

Finally $\left(\mathbf{M}_{i}\right)_{i=0, . ., n}$ is a set of $3 \mathrm{D}$ points representing the shape of the deformed beam. It should be noted that the resolution can be generalized to discrete set with non constant step by using $h_{i}=s_{i+1}-s_{i}$ instead of $h$ in (21) to (28).

\section{Materials}

This section describes the beam instrumentations used and the test bench designed.

\subsection{Beam Characteristics}

The material chosen for beams is fiberglass. The main reasons for this choice is that fiberglass is flexible, so that deformations can be done easily, and that it is compatible with the imaging modalities used to recover the deformed shape of the beam. As a non-ferromagnetic material, fiberglass will not produce any effect during the computerized tomography (CT) acquisitions. Thus, the beams considered are fiberglass cylindrical hollow tubes of $450 \mathrm{~mm}$ length with an outer radius of $3 \mathrm{~mm}$ and an inner radius of $1 \mathrm{~mm}$. 


\subsection{Beam Instrumentation}

The strain gauges are fixed on the beam parallel to the beam direction, as shown in figure 5 , in order to measure the beam axial surface strain. They have a

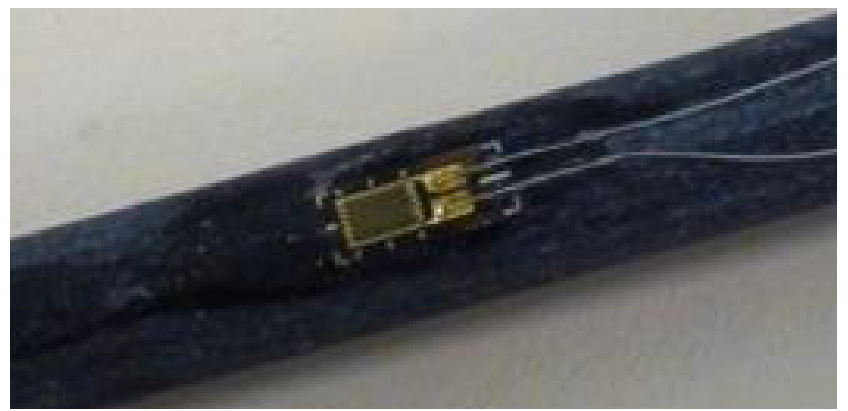

Fig. 5 Strain gauge fixed on the beam.

length of $3 \mathrm{~mm}$ and a width of $2 \mathrm{~mm}$. Thus, the value of the strain gauge semi-angle $\beta$, as defined in figure $3 \mathrm{a}$, is:

$\beta \approx 19^{\circ}$

According to figure 4 , this means that the curvature value is underestimated by approximately $2 \%$ by the IW model compared to the FW model. The strain gauges have a resistance value of $120 \mathrm{ohms}$ and a gauge factor of 2. The bonding of the strain gauges on the beam was realized with a glue designed for fiberglass. An acquisition system was developed to retrieve strain gauges values in real-time. As this process involves sensing extremely small resistance variations, Wheatstone bridges are used in order to measure accurately the resistance changes. The output signals of the Wheatstone bridges are then amplified by a signal conditioner (model Seneca Z-SG Strain Gauge Converter) before being dispatched to the data acquisition card of a PC. The experimental set-up of the acquisition system is presented in figure $6 \mathrm{a}$. The resistance signals are then processed by the $\mathrm{PC}$ which computes the strain of each gauges in real-time, as shown in figure $6 \mathrm{~b}$.

\subsection{Test Bench}

In order to evaluate the beam shape reconstruction accuracy, a test bench has been designed to compare the shape of a beam deformed experimentally and its reconstruction from strain gauges data. The bench is composed of a beam clamped to the side of the box, as shown in figure 7 . The beam has been instrumented with two strain gauges triplets as illustrated in figure $3 \mathrm{~b}$.

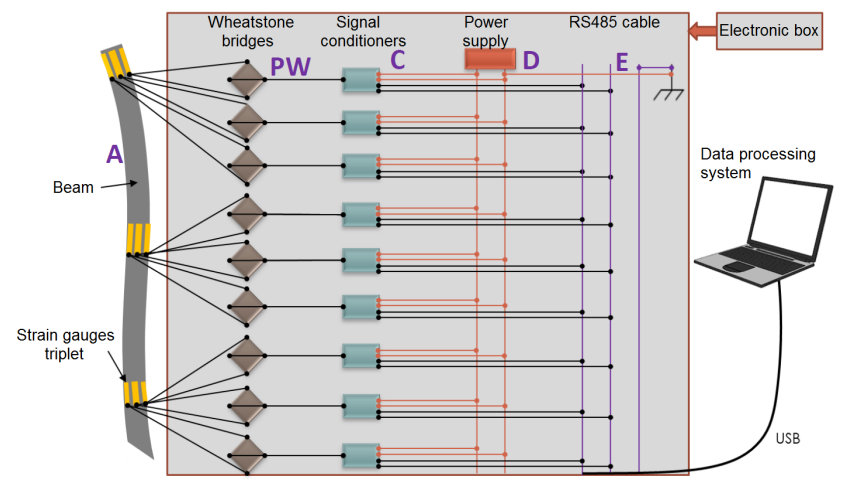

(a) Acquisition process of the strain gauges signals.

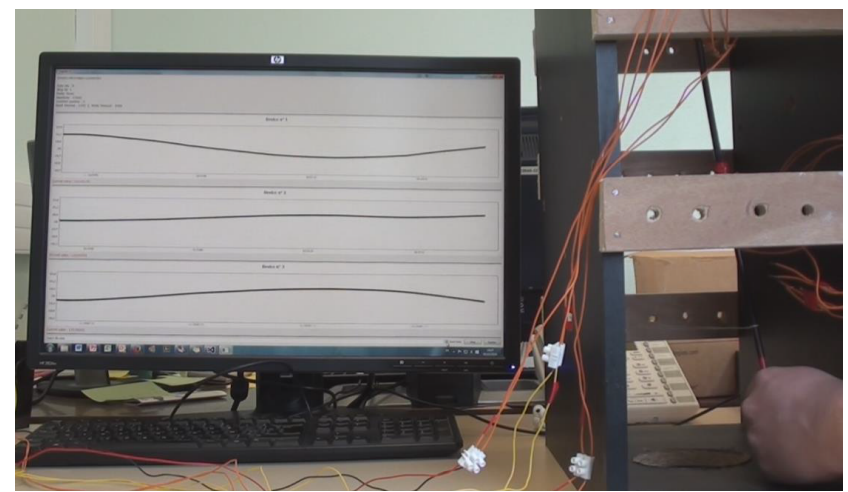

(b) Real-time monitoring of the strain gauges resistance values.

Fig. 6 Strain gauges acquisition system

The problem of strain sensor positioning on beams with circular cross sections has been adressed by Park et al. [4] and Abayazid et al. [27] in their works on needles. In these works, Park et al. and Abayazid et al. define the strain sensor positioning problem as a minimization problem whose solutions are the positions minimizing the errors of reconstruction of the deformed needle shapes. The deformed needles shapes used are created from arbitrary load cases. The more general question of the optimal sensors positions has also been addressed in the context of continuum robotics by Kim et al. [34] and Mahoney et al. [35] specifically for concentric-tube robots. In Kim et al. the sensors are placed to minimize the reconstruction error of the robot shape which is represented as a linear combination of spatial functions. In their work, Mahoney et al. have shown that the shape reconstruction and sensor positioning are coupled problems which means that the best position does not only depend of the parameters chosen for the minimization but also of the reconstruction method. Consequently, the optimal position obtained with a certain reconstruction method can be not legitimate for an other reconstruction method. For these reasons, the optimal triplets locations used here are taken from Schaefer et 

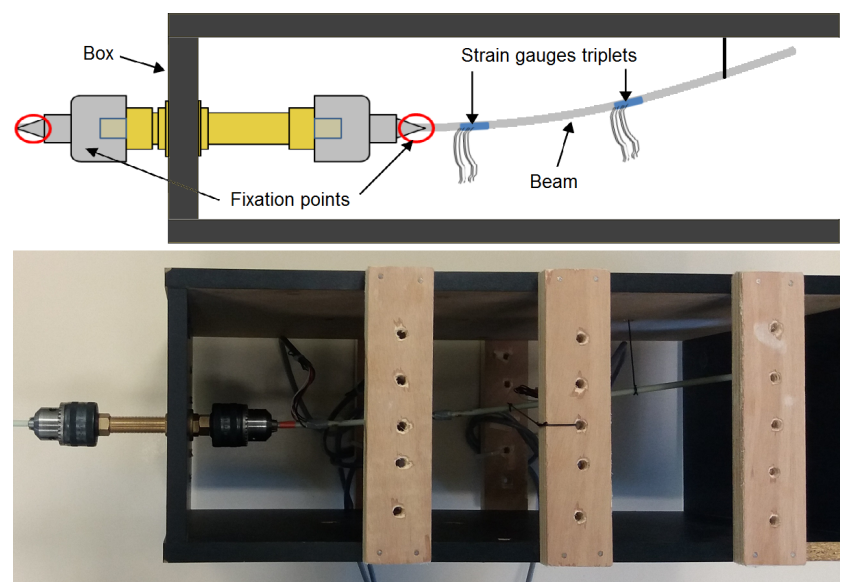

Fig. 7 Beam deformation bench: Deformed position: A cable is attached to the beam near its extremity and is fixed to the box. The beam undergoes a deformation.

al. [29] as the reconstruction method in this work is identical to the method presented here. These locations refers to optimal locations for strain sensors on needles, but because it has been computed with the same reconstruction method from real experimental deformations we believe that it will give relevant triplets locations. Fitting the results provided in Schaefer et al. to the current beam length then gives triplets locations of 45 $\mathrm{mm}$ and $200 \mathrm{~mm}$ from the beam fixation.

\section{Methods}

This section describes the specifications of the beam experiment developed to validate the reconstruction process. In order to control the deformation applied on the beam of the test bench, cables are fixed to the beam and then hooked to one of the several attachment points disposed on the sides of the box, as shown in figure 7 . For each of the deformation, a CT-scan of the bench had been realized and the strain gauges data have been acquired. Deformations 1 and 2 are the results of two orthogonal loads applied on the beams whereas a single load was applied in case of deformations 3 and 4 . Thus, in case of deformations 1 and 2 the beam has a 3D shape whereas the deformations 3 and 4 are planar. The deformations characteristics of the beams are presented in table 1. Results show that the beam undergoes large displacements in case of deformations 2 , 3 and 4 as the deflections are greater than $10 \%$ of the beam length.

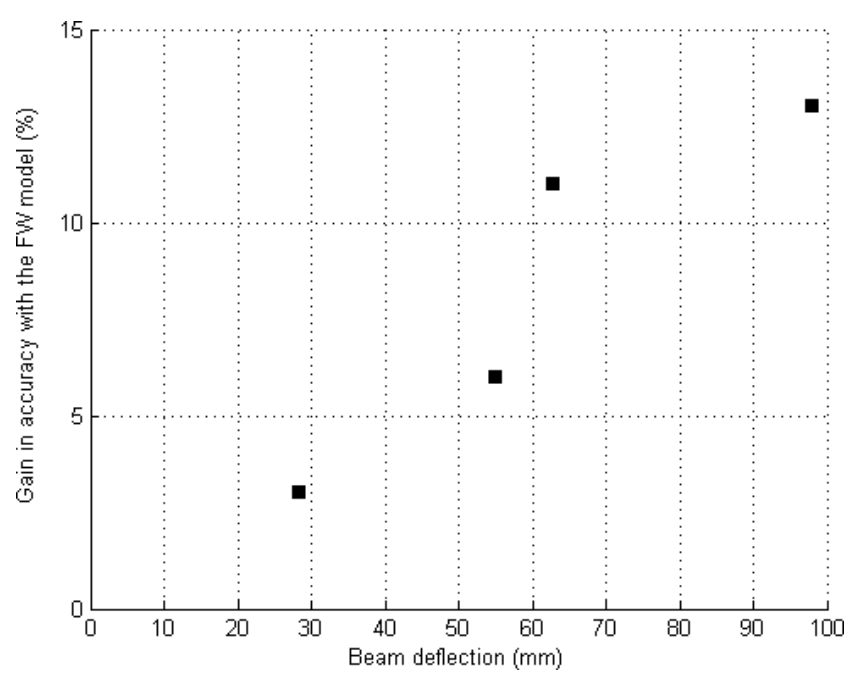

Fig. 8 Gain in reconstruction accuracy using the FW model.

\section{Results}

The deformed beam shapes have been retrieved from scanner images by segmentation and registration. The strain acquisitions from the two triplets were used to recover the curvature and bending angle values at the triplet locations. These values were then interpolated linearly on the whole length of the beam using null curvature constraint at the end of the beam. From the resulting curvature and bending angle estimates were reconstructed the beam shapes with the method presented in Section 2.2 using a step value $\mathrm{h}=1 \mathrm{~mm}$. The beam shapes were reconstructed using both IW and FW gauge model for comparison purposes.

One of main criteria to evaluate the accuracy of the reconstruction is the distance between the tip of the beam shape and the tip of the reconstructed shape of the beam $[13,19,15,4,5]$. This distance is called tip error. The tip errors results of the reconstructions are presented in table 2. For the shape reconstruction of deformation 1 and 2, the tip errors are $4.8 \mathrm{~mm}$ and $12.9 \mathrm{~mm}$ with the IW model and $4.7 \mathrm{~mm}$ and $12.0 \mathrm{~mm}$ with the FW model. For the shape reconstruction of deformation 3 and 4, the tip errors are $7.9 \mathrm{~mm}$ and $8.3 \mathrm{~mm}$ with the IW model and $7.0 \mathrm{~mm}$ and $7.3 \mathrm{~mm}$ with the FW model. Thus, the tip errors of the four experimental deformations are smaller when the beam shape is reconstructed using the FW model instead of the IW model. The original beam shapes and their reconstructed shapes with the FW model are presented conjointly for each deformation in figure 9 and figure 10. For deformations 1 and 2, with respective beam deflections of $28.5 \mathrm{~mm}$ and $55.0 \mathrm{~mm}$, the gain in accuracy with the FW model is $3 \%$ and $6 \%$ and for deformations 
Table 1: Beam characteristics for deformations 1 to 4 .

\begin{tabular}{|c|c|c|c|}
\hline Deformation & Beam length $(\mathrm{mm})$ & Beam loads position & Deflection $(\mathrm{mm})$ \\
\hline 1 & 450 & $250 \mathrm{~mm}(\mathrm{Oz}) / 400 \mathrm{~mm}(\mathrm{Oy})$ & 28.5 \\
\hline 2 & 450 & $250 \mathrm{~mm}(\mathrm{Oz}) / 400 \mathrm{~mm}(\mathrm{Oy})$ & 55.0 \\
\hline 3 & 450 & $400 \mathrm{~mm}(\mathrm{Oy})$ & 62.9 \\
\hline 4 & 450 & $400 \mathrm{~mm}(\mathrm{Oy})$ & 98.1 \\
\hline
\end{tabular}

Table 2: Beam reconstruction characteristics. $E_{I W}$ and $E_{F W}$ are the reconstruction tip errors for the strain gauge models IW and FW. The gain in reconstruction accuracy with the FW strain gauge model is expressed in absolute terms and relatively to $E_{I W}$.

\begin{tabular}{|c|c|c|c|c|}
\hline Deformation & $E_{I W}(\mathrm{~mm})$ & $E_{F W}(\mathrm{~mm})$ & Gain in accuracy $(\mathrm{mm})$ & Gain in accuracy $\left(\%\right.$ of $\left.E_{I W}\right)$ \\
\hline 1 & 4.8 & 4.7 & 0.1 & $3 \%$ \\
\hline 2 & 12.9 & 12.0 & 0.9 & $6 \%$ \\
\hline 3 & 7.9 & 7.0 & 0.9 & $11 \%$ \\
\hline 4 & 8.3 & 7.3 & 1.0 & $13 \%$ \\
\hline
\end{tabular}

Table 3: Comparison of beam reconstruction characteristics of the experimental deformations with the results of Gu et al. [36] and Payo et al. [37]. Deflection and tip error E are given as relative values for comparison purposes.

\begin{tabular}{|c|c|c|c|c|}
\hline Experiment & Deflection (\% of Beam length) & Cross sections instrumented & Strain measures & $E$ (\% of Deflection) \\
\hline Gu et al. & $3 \%$ & 4 & 16 & $7 \%$ \\
\hline Payo et al. & $3 \%$ & 4 & 10 & $12 \%$ \\
\hline Deformation 1 & $6 \%$ & 2 & 6 & $16 \%$ \\
\hline Deformation 2 & $12 \%$ & 2 & 6 & $22 \%$ \\
\hline Deformation 3 & $14 \%$ & 2 & 6 & $11 \%$ \\
\hline Deformation 4 & $22 \%$ & 2 & 6 & $7 \%$ \\
\hline
\end{tabular}

3 and 4 whose beam deflections are $62.9 \mathrm{~mm}$ and 98.1 $\mathrm{mm}$ the gain is $11 \%$ and $13 \%$. Therefore the larger the beam deflection is, the bigger the gain in accuracy is. The gain in accuracy according to the beam deflection is presented in figure 8. The tip error of the deformations increases with the deflection of the beam except for deformation 2. In fact the tip error of deformation 2 is bigger than the tip error of deformations 3 and 4 who have higher deflections. This can be explained by the nature of deformation 2 which combines large displacement and 3D aspect. The 3D aspect in particular seems to play an important role as the the tip errors of the $3 \mathrm{D}$ deformations 1 and 2 represent $16 \%$ and $22 \%$ of the beam deflections compared to $11 \%$ and $7 \%$ for the $2 \mathrm{D}$ deformations 3 and 4 as shown in table 3 .

\section{Discussion}

In this study, a novel model taking into account the width of the gauge was presented. This model is useful when the strain field of the surface on which the gauge is fixed is not uniform. This is the case for the deformations of beam structures presented in this paper. Results show that using the FW model improves the reconstruction accuracy compared to the IW model. This reconstruction accuracy improvement brought by the FW model increases with the beam deflection. In fact, as beams with the largest deflection have also the bigger curvature value, the larger the deflection is, the bigger the curvature correction made by the FW model will be.

The number of sensors and their configurations on the beam constitute input parameters of the beam shape monitoring. The configurations of the sensors include the locations of the triplets on the beam and the angles where the sensors are positioned around the cross section. The accuracy with which the sensors are positioned on the beam can then be a potential source of error for these input parameters and is then a factor influencing the accuracy of the shape monitoring. A sensitivity analysis of circular beams instrumented with strain sensors has been performed by Henkel et al. [15] in work concerning needles instrumented with fiber Bragg gratings. In this work, the instrumented needle was modelized by a beam with triplets of strain sensors positioned at $120^{\circ}$. Besides the difference of sensor technology, the results presented are thus still relevant with our work. Using simulations Henken et al. showed that the error in the estimate of curvature and bending angle is linearly related to the angular position inaccuracy around the cross section. The maximal error for an innacurate positioning of $2^{\circ}$ is approximately $3 \%$ of the curvature and $2^{\circ}$ for the bending angle. Concern- 

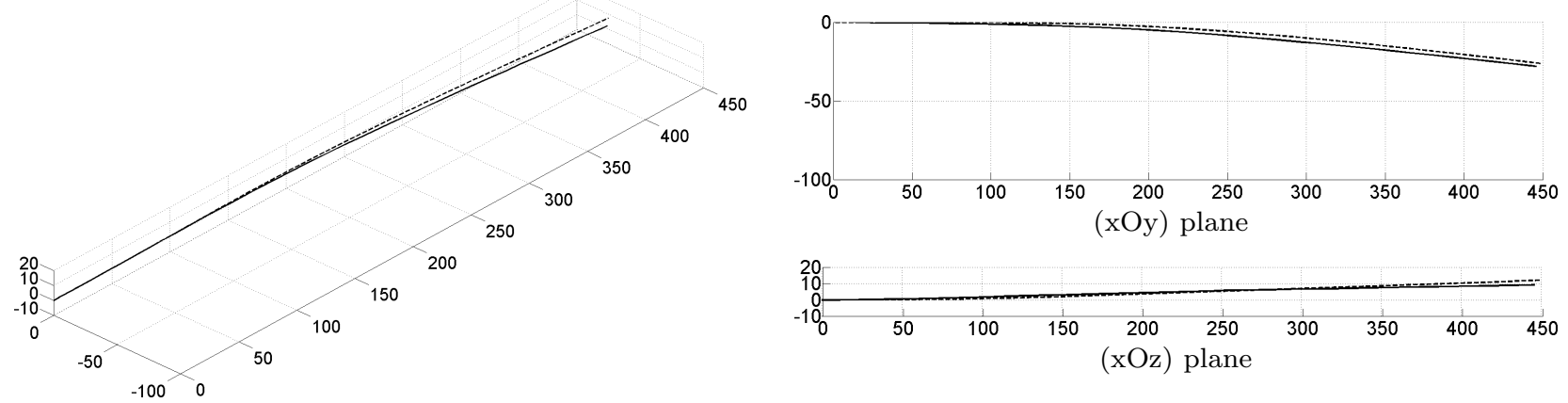

(a) Deformation 1 : the beam deflection is $28.5 \mathrm{~mm}$ which corresponds to $6 \%$ of the beam length.
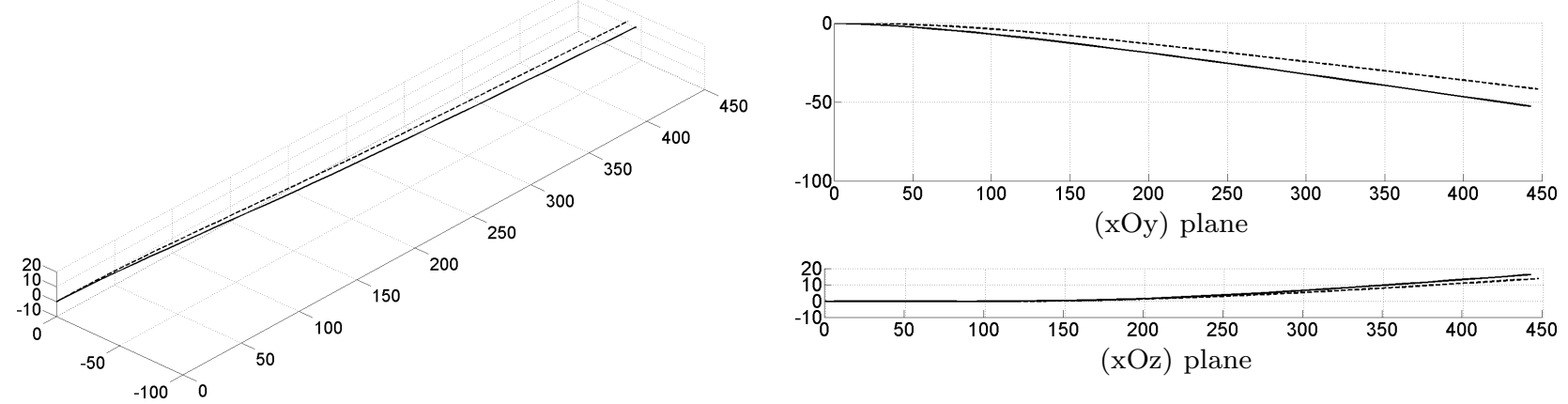

(b) Deformation 2 : the beam deflection is $55.0 \mathrm{~mm}$ which corresponds to $12 \%$ of the beam length.

Fig. 9 Original shape (plain) and reconstructed shape (dotted) of a 3D deformed beam. Loads are located at 250 $\mathrm{mm}$ and $400 \mathrm{~mm}$ directed towards the $(\mathrm{Oz})$ and $(\mathrm{Oy})$ axis. Units are in millimeters.

ing the impact of triplets locations, simulation results show that for a number of triplet comprised between one to five, the maximum error due to triplets positioning inaccuracy of $2 \mathrm{~mm}$ for a $200 \mathrm{~mm}$ needle is $6 \%$ of the reconstruction error. These results were based on simulated shapes obtained from 2D distributed forces loads which were also used to evaluate the impact of the number of triplets on the reconstruction accuracy. It was shown that unsurprisingly the accuracy of shape sensing increases when a triplet is added, the effect becoming small when used in excess of five triplets. The combined effect of these inaccuracies in sensor configurations (locations and angular positions) gives a maximal error in shape estimation of $7.1 \%$ of the reconstruction error. The actual sensitivity of the shape sensing to the input parameters is hard to assess as it depends of multiple factors such as beam deformations and cannot be predicted beforehand. Nevertheless, the results of Henken et. al. show that the gain in accuracy provided by the use of the FW model (up to $13 \%$ ) is not negligible compared to error caused by sensor positioning inaccuracy.

Papers in literature dealing with beam reconstruction from strain gauges present tip errors results of experimental reconstructions for beam structures with different length and deflections. These results can then be compared by expressing the tip errors as a percentage of the deflection. Thus, $\mathrm{Gu}$ et al. [36] presents the reconstruction of a $138 \mathrm{~cm}$ flexible arm with a tip error of $2.9 \mathrm{~mm}$ for a $42.7 \mathrm{~mm}$ deflection, which corresponds to an error of $7 \%$ of the deflection. In their work, Payo et al. [37] reconstruct a $120 \mathrm{~cm}$ beam with a tip error of $3.8 \mathrm{~mm}$ for a $32 \mathrm{~mm}$ deflection, which corresponds to an error of $12 \%$ of the deflection. The results of deformations 3 and 4 which are $11 \%$ and $7 \%$ are consistent with these tip errors results. The tip errors of deformation 1 and 2 are higher with values of $16 \%$ and $22 \%$ of the beam deflection. The higher values of these errors can be explained by the 3D property of these deformations, implying that two strain triplets are not enough to capture all their complexities thus leading to less accurate reconstructions. Consequently the less good results obtained in those two cases come from the beam being instrumented with only six strain sensors. Nevertheless, to put these results into perspective it should be noted that the reconstruction results of $\mathrm{Gu}$ et al. have been obtained with 16 strain measures and the results of Payo et al. with 10 strain measures. Furthermore, in both works of Payo et al. and $\mathrm{Gu}$ et al. only small displacement deformations were considered, the value 

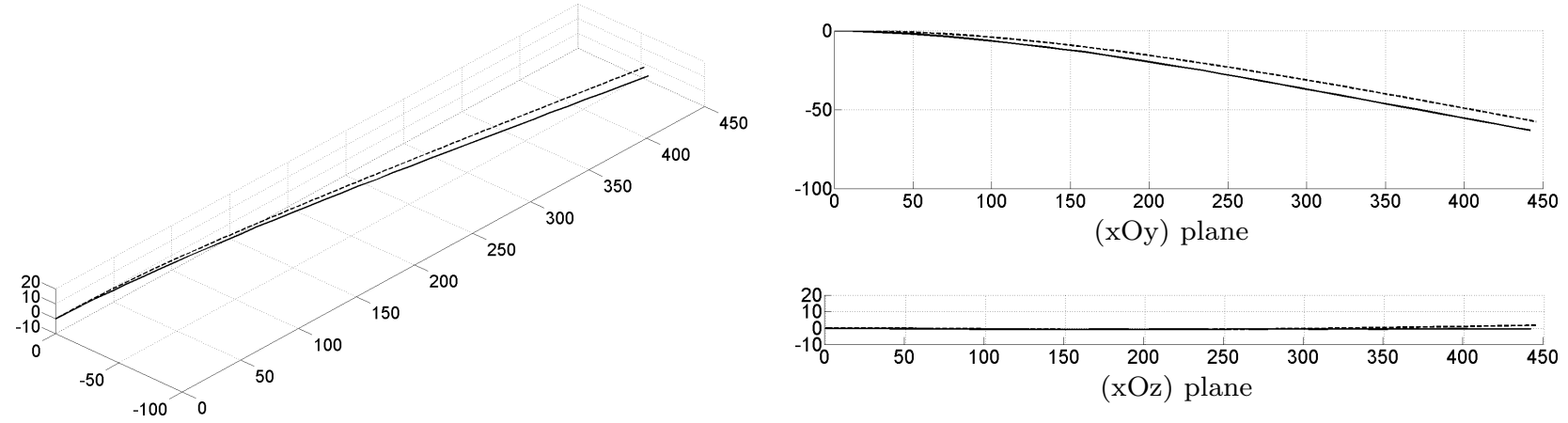

(a) Deformation 3 : the beam deflection is $62.9 \mathrm{~mm}$ which corresponds to $14 \%$ of the beam length.
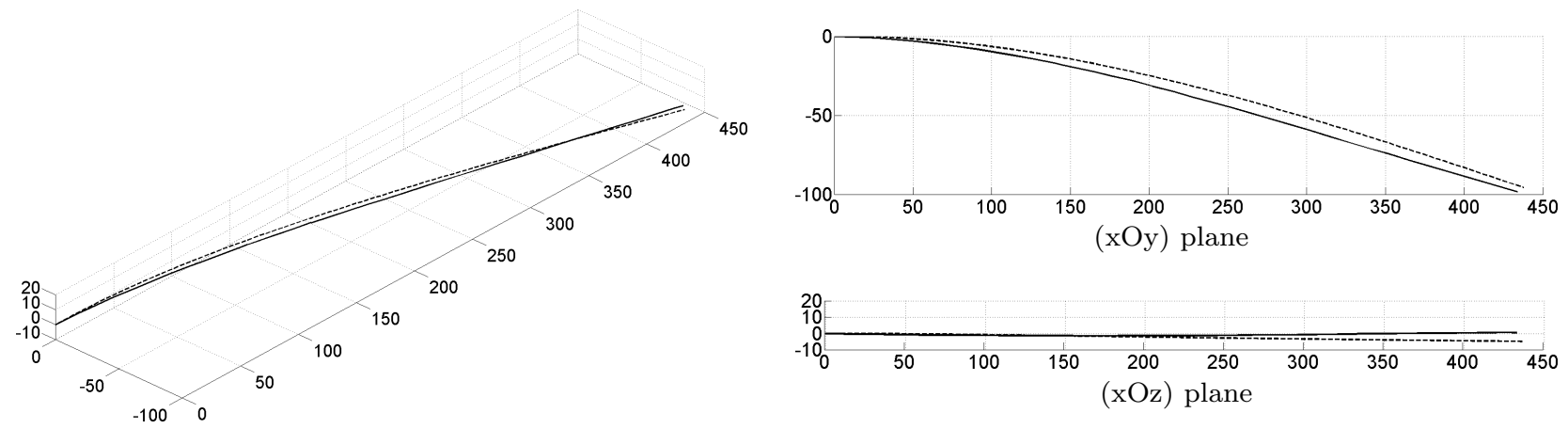

(b) Deformation 4 : the beam deflection is $98.1 \mathrm{~mm}$ which corresponds to $22 \%$ of the beam length.

Fig. 10 Original shape (plain) and reconstructed shape (dotted) of a 2D deformed beam. Loads are located at $400 \mathrm{~mm}$ directed towards the (Oy) axis. Units are in millimeters.

of the beam deflection being approximately $3 \%$ of the beam length whereas the deformations considered in our work include larger deflections from $6 \%$ to $22 \%$ of the beam length, as shown in table 3 . Consequently, despite the large deflections of the beams considered, the beam shape reconstruction results presented in this paper obtained from a limited number of strain measures are consistent with the literature. These results demonstrate the ability of the novel techniques presented in this article to achieve the goal of monitoring beam deformed shapes correctly.

Differents improvements could be performed on the beam instrumentation in order to increase the beam shape reconstruction accuracy. In fact, as mentionned previously, changing the number of strain sensors by adding additional strain gauge triplets on the beam would be an effective way to improve the reconstruction accuracy in case of 3D deformations. Interestingly, the number of strain triplets is not the only change that could be considered to improve the reconstruction accuracy. The positioning of the strain sensors parallel to the beam thus allows to have access to the bending informations of the beam. By changing the direction of the strain sensors to another direction, not parallel to the beam, it would be possible to obtain informations related to the torsion of the beam. Future work will focus on the development of a model of beam instrumented with strain gauges able to capture both axial and shear strain and how to use these to improve the reconstruction accuracy.

\section{Conclusion}

A 3D shape monitoring system for beam with strain gauges has been presented. The strain gauges were fixed on the surface of the beam by groups of three on the same cross section. An acquisition system was developed to measure the strain at each gauge locations. A finite width strain gauge model was presented in order to recover the estimates of the curvature and the bending angle of the beam while taking into account physical characteristics of 3D deformations. These estimates were used as inputs of the 3D finite strain large displacement beam shape reconstruction method to approximate the deformed beam shape. Results showed that correct beam shape reconstructions were achieved with only a limited number of strain measures, demonstrating the effectiveness of beam instrumentation, the strain gauge model and the reconstruction method pro- 
posed. Moreover, the creation of a finite width gauge model is not restricted to beams with circular cross section as the technique of strain field averaging over gauge width can be applied to any cross section geometry.

The approach and model presented here are particularly suitable for applications monitoring beam undergoing 3D deformations with strain gauges of significant size compared to the structure. In the medical field for instance, needle shape monitoring from strain gauges, such as in Hammond et al. [38] and Bonvilain et al. [39] is a potential domain of application for the work presented in this paper.

\section{Conflicts of interest}

On behalf of all authors, the corresponding author states that there is no conflict of interest.

\section{Acknowledgment}

This work is part of the project GAME-D, financed by the French National Agency for Research (ref: ANR12-TECS-0019) and supported by Laboratory of Excellence CAMI (ref: ANR-11-LABX-0004-01).

The authors would like to thank Cecilia Hughes for English corrections and P. A. Barraud for providing valuable advice concerning electronic instrumentation.

\section{References}

1. Abdel-Jaber H, Glisic B (2015) Analysis of the status of pre-release cracks in prestressed concrete structures using longgauge sensors. Smart Materials and Structures 24(2), URL http://stacks.iop.org/0964$1726 / 24 / \mathrm{i}=2 / \mathrm{a}=025038$

2. Lee JJ, Shinozuka M (2006) Real-time displacement measurement of a flexible bridge using digital image processing techniques. Experimental Mechanics 46(1):105-114, DOI 10.1007/s11340-0066124-2, URL https://doi.org/10.1007/s11340-0066124-2

3. Xia Y, Zhang $\mathrm{P}$, qing $\mathrm{Ni} \mathrm{Y}$, ping Zhu $\mathrm{H}$ (2014) Deformation monitoring of a super-tall structure using real-time strain data. Engineering Structures 67(Supplement C):29 - 38, DOI https://doi.org/10.1016/j.engstruct.2014.02.009

4. Park YL, Elayaperumal S, Daniel B, Ryu SC, Shin M, Savall J, Black R, Moslehi B, Cutkosky M (2010) Real-time estimation of $3-\mathrm{d}$ needle shape and deflection for mri-guided interventions 15(6):906-915, DOI 10.1109/TMECH.2010.2080360

5. Roesthuis R, Kemp M, van den Dobbelsteen J, Misra S (2014) Three-dimensional needle shape reconstruction using an array of fiber bragg grating sensors 19(4):1115-1126, DOI 10.1109/TMECH.2013.2269836

6. Lehmann T, Rossa C, Usmani N, Sloboda RS, Tavakoli M (2016) A real-time estimator for needle deflection during insertion into soft tissue based on adaptive modeling of needle. IEEE/ASME Transactions on Mechatronics 21(6):2601-2612, DOI 10.1109/TMECH.2016.2598701

7. Logozzo S, Kilpela A, Makynen A, Zanetti EM, Franceschini G (2014) Recent advances in dental optics part ii: Experimental tests for a new intraoral scanner. Optics and Lasers in Engineering 54:187 - 196, DOI https://doi.org/10.1016/j.optlaseng.2013.07.024

8. Cali M, Oliveri SM, Ambu R, Fichera G (2018) An integrated approach to characterize the dynamic behaviour of a mechanical chain tensioner by functional tolerancing. Strojniski Vestnik/Journal of Mechanical Engineering 64(4)

9. Yan X, Huang W, Kwon SR, Yang S, Jiang $\mathrm{X}$, Yuan FG (2013) A sensor for the direct measurement of curvature based on flexoelectricity. Smart Materials and Structures 22(8):085016, URL http://stacks.iop.org/0964$1726 / 22 / \mathrm{i}=8 / \mathrm{a}=085016$

10. Glaser R, Caccese V, Shahinpoor M (2012) Shape monitoring of a beam structure from measured strain or curvature. Experimental Mechanics 52(6):591-606, DOI 10.1007/s11340-011-9523-y, URL https://doi.org/10.1007/s11340-011-9523-y

11. Cheng B, Zhu W, Liu J, Yuan L, Xiao H (2017) 3d beam shape estimation based on distributed coaxial cable interferometric sensor. Smart Materials and Structures 26(3), URL http://stacks.iop.org/0964$1726 / 26 / \mathrm{i}=3 / \mathrm{a}=035017$

12. Todd MD, Stull CJ, Dickerson M (2013) A local material basis solution approach to reconstructing the three-dimensional displacement of rod-like structures from strain measurements. Journal of Applied Mechanics 80(4)

13. Gherlone M, Cerracchio P, Mattone M, Sciuva MD, Tessler A (2014) An inverse finite element method for beam shape sensing: theoretical framework and experimental validation. Smart Materials and Structures 23(4), URL http://stacks.iop.org/0964$1726 / 23 / \mathrm{i}=4 / \mathrm{a}=045027$

14. Chadha M, Todd MD (2017) A generalized approach for reconstructing the three-dimensional 
shape of slender structures including the effects of curvature, shear, torsion, and elongation. Journal of Applied Mechanics 84(4):041003

15. Henken KR, Dankelman J, van den Dobbelsteen JJ, Cheng LK, van der Heiden MS (2014) Error analysis of fbg-based shape sensors for medical needle tracking 19(5):1523-1531, DOI 10.1109/TMECH.2013.2287764

16. Sigurdardottir DH, Stearns J, Glisic B (2017) Error in the determination of the deformed shape of prismatic beams using the double integration of curvature. Smart Materials and Structures 26(7), URL http://stacks.iop.org/0964$1726 / 26 / \mathrm{i}=7 / \mathrm{a}=075002$

17. Wang ZC, Geng D, Ren WX, Liu HT (2014) Strain modes based dynamic displacement estimation of beam structures with strain sensors. Smart Materials and Structures 23(12), URL http://stacks.iop.org/0964$1726 / 23 / \mathrm{i}=12 / \mathrm{a}=125045$

18. Kim NS, Cho NS (2004) Estimating deflection of a simple beam model using fiber optic bragg-grating sensors. Experimental Mechanics 44(4):433-439, DOI 10.1007/BF02428097, URL https://doi.org/10.1007/BF02428097

19. Moon H, Jeong J, Kang S, Kim K, Song YW, Kim J (2014) Fiber-bragg-grating-based ultrathin shape sensors displaying single-channel sweeping for minimally invasive surgery. Optics and Lasers in Engineering 59:50 - 55, DOI http://dx.doi.org/10.1016/j.optlaseng.2014.03.005

20. Wang H, Zhang R, Chen W, Liang X, Pfeifer R (2016) Shape detection algorithm for soft manipulator based on fiber bragg gratings. IEEE/ASME Transactions on Mechatronics 21(6):2977-2982, DOI 10.1109/TMECH.2016.2606491

21. Childlers B, Gifford D, Duncan R, Raum M, Vercellino M (2006) Fiber optic position and shape sensing device and method relating thereto. US Patent App. 11/180,389

22. Xu R, Yurkewich A, Patel RV (2016) Curvature, torsion, and force sensing in continuum robots using helically wrapped fbg sensors. IEEE Robotics and Automation Letters 1(2):1052-1059, DOI 10.1109/LRA.2016.2530867

23. Xu H, Ren WX, Wang ZC (2015) Deflection estimation of bending beam structures using fiber bragg grating strain sensors. Advances in Structural Engineering 18(3):395-403

24. Reissner E (1973) On one-dimensional largedisplacement finite-strain beam theory. Studies in Applied Mathematics 52(2):87-95
25. Ryu SC, Dupont PE (2014) Fbg-based shape sensing tubes for continuum robots. In: 2014 IEEE International Conference on Robotics and Automation (ICRA), pp 3531-3537, DOI 10.1109/ICRA.2014.6907368

26. Hoffmann K (1989) An introduction to measurements using strain gages. Hottinger Baldwin Messtechnik Darmstadt

27. Abayazid M, Kemp M, Misra S (2013) 3d flexible needle steering in soft-tissue phantoms using fiber bragg grating sensors. In: Proc. IEEE International Conference on Robotics and Automation (ICRA), pp 5843-5849, DOI 10.1109/ICRA.2013.6631418

28. Schajer GS (1993) Use of displacement data to calculate strain gauge response in non-uniform strain fields. Strain 29(1):9-13, DOI 10.1111/j.14751305.1993.tb00820.x

29. Schaefer PL, Chagnon G, Moreau-Gaudry A (2016) Advanced sensors placement for accurate $3 \mathrm{~d}$ needle shape reconstruction. In: Engineering in Medicine and Biology Society (EMBC), 2016 IEEE 38th Annual International Conference of the, IEEE, pp 5132-5135

30. Wang W, Jüttler B, Zheng D, Liu Y (2008) Computation of rotation minimizing frames. ACM Transactions on Graphics (TOG) 27(1):2

31. Hairer E, Wanner G, Lubich C (2006) Geometric Numerical Integration. Structure-Preserving Algorithms for Ordinary Differential Equations, 2nd edn

32. Magnus W (1954) On the exponential solution of differential equations for a linear operator. Communications on Pure and Applied Mathematics 7(4):649-673, DOI 10.1002/cpa.3160070404, URL http://dx.doi.org/10.1002/cpa.3160070404

33. Simo J, Fox D (1989) On a stress resultant geometrically exact shell model. part i: Formulation and optimal parametrization. Computer Methods in Applied Mechanics and Engineering 72(3):267

304, DOI http://dx.doi.org/10.1016/00457825(89)90002-9

34. Kim B, Ha J, Park FC, Dupont PE (2014) Optimizing curvature sensor placement for fast, accurate shape sensing of continuum robots. In: 2014 IEEE International Conference on Robotics and Automation (ICRA), pp 5374-5379, DOI 10.1109/ICRA.2014.6907649

35. Mahoney AW, Bruns TL, Swaney PJ, Webster RJ (2016) On the inseparable nature of sensor selection, sensor placement, and state estimation for continuum robots or where to put your sensors and how to use them. In: Robotics and Automation (ICRA), 2016 IEEE International Conference on, IEEE, pp 4472-4478 
36. Gu M, Piedbuf JC (2003) A flexible-arm as manipulator position and force detection unit. Control Engineering Practice 11(12):1433 - 1448, DOI https://doi.org/10.1016/S0967-0661(03)00105-9, award winning applications-2002 IFAC World Congress

37. Payo I, Feliu V (2014) Strain gauges based sensor system for measuring 3-d deflections of flexible beams. Sensors and Actuators A: Physical 217(Supplement C):81 - 94, DOI https://doi.org/10.1016/j.sna.2014.06.014

38. Hammond FL, Smith MJ, Wood RJ (2014) Estimating surgical needle deflection with printed strain gauges. In: 2014 36th Annual International Conference of the IEEE Engineering in Medicine and Biology Society, pp 6931-6936, DOI 10.1109/EMBC.2014.6945222

39. Bonvilain A, Gangneron M (2016) Characterization of strain microgauges for the monitoring of the deformations of a medical needle during its insertion in human tissues. Microsystem Technologies 22(3):551-556, DOI 10.1007/s00542-015-25882, URL https://doi.org/10.1007/s00542-015-2588-2 